\title{
Diagnosis of hypercritical chronic pulmonary disorders using dense convolutional network through chest radiography
}

\author{
Rajat Mehrotra $^{1}$ (D) Rajeev Agrawal ${ }^{2}$ - M. A. Ansari ${ }^{3}$
}

Received: 19 May 2021 / Revised: 30 August 2021 / Accepted: 22 November 2021 /

Published online: 28 January 2022

(c) The Author(s), under exclusive licence to Springer Science+Business Media, LLC, part of Springer Nature 2022

\begin{abstract}
Lung-related ailments are prevalent all over the world which majorly includes asthma, chronic obstructive pulmonary disease (COPD), tuberculosis, pneumonia, fibrosis, etc. and now COVID-19 is added to this list. Infection of COVID-19 poses respirational complications with other indications like cough, high fever, and pneumonia. WHO had identified cancer in the lungs as a fatal cancer type amongst others and thus, the timely detection of such cancer is pivotal for an individual's health. Since the elementary convolutional neural networks have not performed fairly well in identifying atypical image types hence, we recommend a novel and completely automated framework with a deep learning approach for the recognition and classification of chronic pulmonary disorders (CPD) and COVIDpneumonia using Thoracic or Chest X-Ray (CXR) images. A novel three-step, completely automated, approach is presented that first extracts the region of interest from CXR images for preprocessing, and they are then used to detects infected lungs X-rays from the Normal ones. Thereafter, the infected lung images are further classified into COVID-pneumonia, pneumonia, and other chronic pulmonary disorders (OCPD), which might be utilized in the current scenario to help the radiologist in substantiating their diagnosis and in starting well in time treatment of these deadly lung diseases. And finally, highlight the regions in the CXR which are indicative of severe chronic pulmonary disorders like COVID-19 and pneumonia. A detailed investigation of various pivotal parameters based on several experimental outcomes are made here. This paper presents an approach that detects the Normal lung X-rays from infected ones and the infected lung images are further classified into COVID-pneumonia, pneumonia, and other chronic pulmonary disorders with an utmost accuracy of $96.8 \%$. Several other collective performance measurements validate the superiority of the presented model. The proposed framework shows effective results in classifying lung images into Normal, COVID-pneumonia, pneumonia, and other chronic pulmonary disorders (OCPD). This framework can be effectively utilized in this current pandemic scenario to help the radiologist in substantiating their diagnosis and in starting well in time treatment of these deadly lung diseases.
\end{abstract}

Keywords Chronic pulmonary disorders · COVID-19 · Pneumonia - Chest X-ray (CXR) · Deep learning $\cdot \mathrm{CNN}$

Rajat Mehrotra

rajjatmehrootra@gmail.com

Extended author information available on the last page of the article 


\section{Introduction}

Nowadays, the adverse impact of changing climatic conditions on human health is rising exponentially. There is a huge expansion in the number of individuals who succumbed to death because of the fast ascent in the number of ailments. The cancerous disease is one of the most significant diseases that had led to the greatest number of deaths in recent years. The WHO articulated that more than 60 million individuals experience the ill effects of chronic obstructive pulmonary disease (COPD) and around 3 million people lose their lives every year, making it worldwide the third foremost reason for deaths in the year 2019-2020. Around 10 million individuals suffer from tuberculosis (TB) and approximately 1.6 million people die of it every year, labelling it to be the utmost deadly infection. Kids under the age group of 5 years and 20-35 age group adults are the one who mostly dies because of tuberculosis. More than $95 \%$ of people die due to tuberculosis in countries with middle and low incomes. About 1.7 million individuals lose their life from cancer in the lungs every year, which make it the most fatal disease. More than 330 million individuals experience the ill effects of asthma, making it the most widely recognized chronic infantile disease which influences $14 \%$ of kids around the world and the graph is still rising. Annually around 7 lakhs, kids face death due to pneumonia of which around $80 \%$ of kids are under 2 years [54]. WHO had recognized lung cancer as the most fatal disease amongst all cancer types. Consequently, the timely detection of such cancers in the lungs is significant for human wellbeing. The danger of lung-related ailments is tremendous, particularly in nations with low and middle incomes, where a huge number of individuals are suffering from pollution. As per the world health organization, around 4 million people lose their lives every year because of air-related infections that include pneumonia and asthma. Consequently, it is important to find a way to diminish air contamination. It is likewise fundamental to execute productive symptomatic frameworks that can help with distinguishing lung infections [34].

The new infectious disease called coronavirus (COVID-19) that emerged from China in late 2019 had now taken the whole world into its grip and has been causing stern damage to the lungs as well as pneumonia and breathing issues [20]. Henceforth, the in-time identification of lung-related diseases has gotten more significant than any time in the recent past. Artificial Intelligence (AI) and deep learning (DL) can perform an imperative part in this. As of late, computer-assisted diagnosis has gotten more significant around the world. Researchers around the globe admit that the DL framework based on chest X-rays could be worthwhile for identifying and evaluating lung diseases. Smart computational techniques for classifying and recognizing diseases have been the key research area for the enhancement of Computer-aided Diagnosis [2, 8, 14, 28]. Resemblances in the manifestations of various diseases may result in medical misinterpretation of actual infections. Such complications are quite recurrent in nations with tropical climatic conditions where communicable diseases on detection show similar manifestations, and therefore the consequential slowdown in the identification of disease and its treatment may cause life-threatening complications for patients of all ages [15]. In the recent times of coronavirus pandemic, cases of misdiagnosis of disease are reported either due to misinterpretation of pulmonary disorders like TB and common pneumonia to COVID infection or due to unavailability of sufficient medical experts and resources. This misinterpretation of disease happens largely due to a lack of sufficient and experienced medical experts and the problem gets intensified in the situations like COVID-19 where there is a large pressure on the medical infrastructure. As the medical infrastructure and shortage of experienced medical practitioners 
cannot be improved instantly there is a need for some intelligent system that can help the medical experts in substantiating their diagnosis and in starting well in time treatment of these deadly diseases. The work presented in this paper aims to give medical experts a heading in the direction of implementing the DL framework for identifying lung-related diseases. The framework introduced here can help the experts to identify the types of lung illnesses very precisely, which can lead to the in-time treatment of the deadly diseases and hence can reduce further complications. Numerous researches had been done to utilize AI techniques for disease identification from chest X-rays [46, 48, 49]. With the help of digital computers, enormous medical records can be managed and the same can be made available to the general population. Such an arrangement can diminish the clinical expenses with the augmentation of computer-aided diagnosis in the health care industry. Hence in this paper, a three-step, completely automated, approach is presented that:

- Firstly, self-extracts the ROI from the input raw image and pre-processes it for image improvement using median filtering. These improved images are then fed into the deep learning system that detects infected lungs X-rays from the Normal ones.

- Secondly, the infected lung images are further classified into COVID-19, pneumonia, and other chronic pulmonary disorders (OCPD), which might give medical experts a lead in the direction of instigating this DL framework for timely detection of lungrelated diseases.

- And finally, it highlights the regions in the CXR which are indicative of severe chronic pulmonary disorders like COVID-19 and pneumonia.

- Also, a detailed investigation of various pivotal parameters such as Cohen's kappa coefficient, Youden's Index, and other collective performance measurements based on the various experimental outcomes is made that further validates the superiority of the presented model.

The rest of the paper is organized as Sect. 2 talks about the related past research work, Sect. 3 will discuss the methodology adopted for the presented research work, Sect. 4 will elaborate on the results of the research, and Sect. 5 will conclude the paper.

\section{Related research work}

Despite the introduction of an initial computer-based diagnosis framework for identifying the infected cells in the lungs during the 1980s, the efforts gone in vain and this is due to the computational structure which was insufficient for the usage in the processing of images. Utilizing the elementary techniques of processing the images is quite tedious. Subsequently, the development of graphics processing units (GPU) and deep learning-based $\mathrm{CNN}$ has improved the working of computer-based diagnosis frameworks. As of now, experts from around the planet, from a varied region of research are working uninterrupted to combat this destructive lung disease. Various investigative explorations have been carried out for demonstrating the strategies and approaches for distinguishing COVID-19 from other chronic pulmonary disorders (CPD) utilizing chest radiographic images. Here we present some pivotal research works done in this area. A 3-dimensional deep convolutional neural network(D-CNN) is proposed in [16] that identifies the nodules in the lungs using image segmentation. Nevertheless, the work by the author had not presented the disease type classification as well as the multiple approaches for disease prediction are applied 
over nodules of small size. The author [43] proposed a completely connected convolutional neural network for reducing the rate of false-positive detection in the classification of nodules in the lungs. Their proposed technique investigates the $888 \mathrm{CT}$ images for reducing the likelihood of a wrong finding.

In [58] R-CNN is utilized for identifying the infected lungs along with the reduction of the rate of false-positive predictions. They designed a 3D R-CNN with three-dimensional twin blocks and an encoder-decoder framework similar to U-Net for detecting the lung nodules. Their proposed technique exhibited anticipated outcomes with $99.04 \%$ accuracy for desired nodule detection. In [33] a computerized COVID identification system is presented to identify normal X-ray images from COVID-19 employing machine learning and deep convolutional based pretrained networks. Their experimental results have accomplished the accuracy of $98.8 \%$ using ResNet50 in identifying normal and COVID infected images. In [41] Frangi filter is used to enhance the detection of nodules from CXR images giving 94\% sensitivity with a rate of false-positive predictions of 15.1. In [24] the importance of AI is elaborated with the best-in-class literature involving the CXR analysis as well as classification. Moreover, their presented work portrays this issue other than maintaining a database known as chestX-ray8 which contains 1,08,948 images out of which more than 32,000 images were of newly detected patients. They also applied D-CNN for result validation on this dataset and achieved the desired results. Authors [22] present a dataset CheXpert that contains 2,24,316 CXR having different image categories of more than 65,000 patients. They applied CNN to mark the imageset on the possibility demonstrated by their model. Their projected model employed frontal as well as lateral CXR images for observation. In $[40,44]$ authors presented a commanding methodology namely FCN and F-RCNN. MaskRCNN (M-RCNN) is an extended version of F-RCNN and is far better than the previous one as far as efficiency and accuracy are concerned.

In [17] authors presented an M-RCNN technique which is a theoretically modest system for segmentation of image objects. Their method proficiently distinguishes the image objects and generating a superior segmentation mask simultaneously for the individual specimen. They contrast their proposed algorithm with that of others to gives the finest algorithm from that in [21, 45]. In [36] a combination of networks (MixNet) is utilized for the identification of nodules occurring in the lungs using two datasets LUNA16 and LIDC-IDRI. The proposed gradient boosting algorithm along with MixNet for classifying the nodules in the images. Their presented framework was tested using contours drawn by the expert radiologist on the lung images including more than 3200 nodules through statistical measures. Their system attained improved results as compared to the standard state of art techniques with sensitivity and specificity of $94 \%$ and $90 \%$ respectively followed by the AUC of 0.99. In [6], and improved AlexNet framework is used to assess irregularities in lung images using two diverse image modalities: X-Ray and lung CT images. Their presented framework is distinctly used over these datasets which initially classifies the X-Ray images into healthy and pneumonia infected with an accuracy of 96.8\%. Moreover, their proposed architecture is also employed for the classification of the CT lung images into benign as well as malignant using both ensembling and non-ensembling of features. Their presented framework with support vector machine as a classifier attains an accuracy of almost 86.5\% and with feature ensembling using comparable DL framework a precision of more than $97 \%$ is achieved. In [7] authors have used the Gabor filter for enhancing the input raw images and Watershed (Marker-Controlled) algorithm for the purpose of segmentation for pointing at the actual region of the infection in addition to this Outof-bag error rate is computed for various iterations as per Random Forest Ensemble 
(RFE) and error rates for the diverse classes are also determined. The highest Out-ofbag error rate is $23.40 \%$ that provides an initial tree. The distribution graph for Mean decrease in accuracy, Gini index, etc. is measured as per the RFE. RUSBoost is finally employed for evaluating the system's accuracy.

In [5] authors designed a system that stores the image information for identifying the consolidation in the pediatric X-ray dataset. They presented a threefold image pre-processing method to augment the model generalization. In order to validate the precision of the mathematical results, they applied an occlusion test for visualizing the outputs of the proposed model as well as localizing the depicted area. They also used another dataset to perform an extra validation to investigate the model generalization. They achieved the best accuracy of $94.67 \%$ in detecting the consolidation which outdoes the other state-of-the-art works by researchers. In [29] authors used a deep learning-based system for detecting pulmonary nodules and masses using four algorithms. They took 100 cases in the investigation and using the above-mentioned algorithms, $76.6 \%$ sensitivity and specificity of $88.68 \%$ are achieved by the mass algorithm in detecting pulmonary nodules and masses at the ideal probability score of 0.2884 as compared to the other three algorithms. Likewise, a DL technique is presented in [10] in which a variety of transfer learning approaches like AlexNet, InceptionV3, DenseNet121, etc. are utilized for the detection of pneumonia by fine-tuning important parameters. Here the image features are extricated using a variety of transfer learning approaches, which are then fed into the classifier. They also used an ensemble model that outpaced the individual pretrained networks and achieves an accuracy of $96.4 \%$.

Authors [25] developed a system for recognizing ribs in CXR images. This system makes use of features based on wavelets apart from different anatomic orientations for classification. In [19] authors focused on the technique of CXR image segmentation, extraction of pivotal features as well as labeling the images as normal and infected depending on the classifier. strategies for division, extraction of highlights, and distinguishing proof of pictures of X-ray as sound and tainted subject to the highlights prepared classifier. In [4] a strategy for the identification of pneumonia is presented by analyzing ultrasound pictures of pneumonia infected lungs and thereafter they are classified using neural networks. In [3] the author presented a system to find and eliminate the skin part considering it as noise from the ultrasound images of the lung to eliminate the misidentification of lungs affected by pneumonia. The author [11] set forward a strategy for recognizing pneumonia using videos of ultrasound imaging. The recordings are analyzed in fragments to create an analysis of the whole video for recognizing pneumonia. In [56] a methodology is proposed dependent on fundamental frequency downshift measurement for distinguishing pneumonia from an image set containing samples of subjects from 6 months to 5 years. Inaccurate diagnosis is the foremost challenge in curing these chronic ailments. Such ailments are repeatedly misinterpreted for other pulmonary infections, like pneumonia, normal cold, etc. [9, 32, 35, 37]. A pulmonary function test is an essential tool for investigating pulmonary fibrosis and COPD, which are hard to do, tedious, and needs a doctor. Infected patients regularly go through computed tomography (CT) examination, which empowers the immediate assessment of lungs using quantitative and visual techniques mutually. Tough, it has numerous restrictions like CT may not be able to detect mild COPD. Likewise, the degree of lung infection might be misjudged due to the indistinctness of localized areas of infection in patients having serious emphysema. 


\section{Implemented methodology}

The prime impetus in doing this research work is to enhance the exactness in identifying and segregating first the infected CXR cases from the normal ones and thereafter classifying the infected images into 3 major categories namely COVID-19, Other CPD's (chronic pulmonary disorder), and pneumonia cases for better understanding and treatment of these disease types. Two different approaches are adopted for this. Model 1 identify and segregate the available CXR images into the infected and normal cases while model 2 further isolates the infected images into above mentioned 3 major categories. The working procedure of the two models is similar where the input image types are first pre-processed for the removal of noise followed by division of the images into training, testing, and validation. $70 \%$ of the available images are kept for training the network while $15 \%$ of images are used individually for both testing and validation of the proposed framework. A fine-tuned DenseNet201deep learning network is utilized here for the proposed detection and classification. Attuning a DL pretrained system like DenseNet201is typically much faster as well as easier rather than starting it to train from very basic. Exploiting a DL framework permits the system to adapt itself to new jobs much quicker. The proposed chronic pulmonary disorder classification models are presented in Fig. 1 and its detailed discussion is presented here.

\subsection{Dataset description and analysis}

A CXR is a normal and cheap clinical imaging method and is high in demand. Though many times it could be quite challenging than chest CT imaging. An ingenious publicly available dataset is lacking and hence, it is extremely testing to acknowledge the medically important analysis and computer-based identification in different clinical destinations utilizing CXR images. The imageset utilized for carrying out the proposed research work is from two public datasets available at $[38,50]$. It consists of around $6000 \mathrm{CXR}$ images, with dimensions $1024 \times 1024$, of different types of pulmonary disorders, out of which, 1949 images are taken for the evaluation of the proposed DL framework. Out of this imageset taken, 1237 are infected lung images and 712 are of normal chest images which are used for the evaluation of model 1 and illustrated in Fig. 2. The infected images are categorized into 9 different classes as elaborated in Fig. 3. The size of all the images is resized to

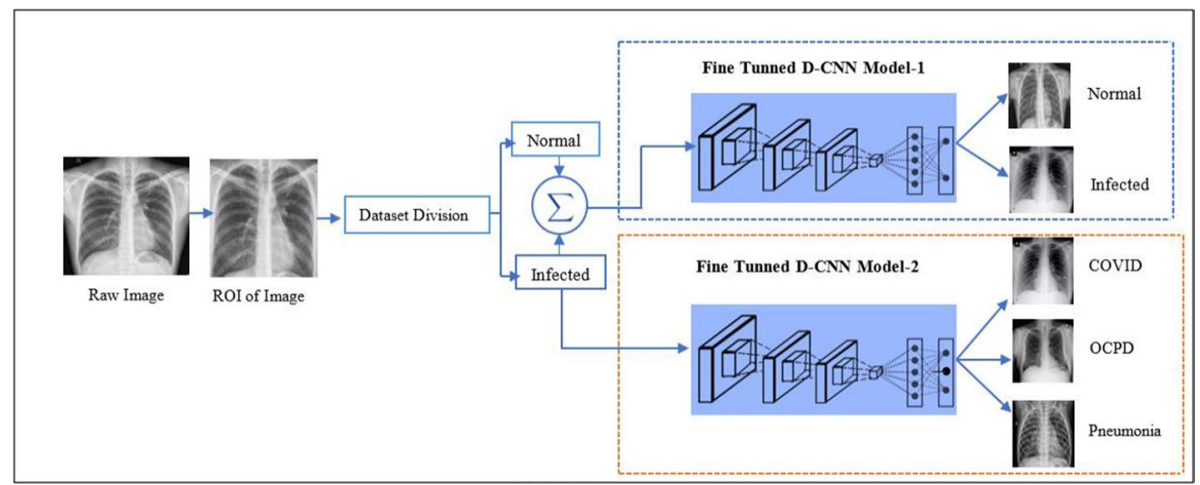

Fig. 1 Proposed pulmonary disorder classification models 
Fig. 2 CXR image distribution of the imageset utilized

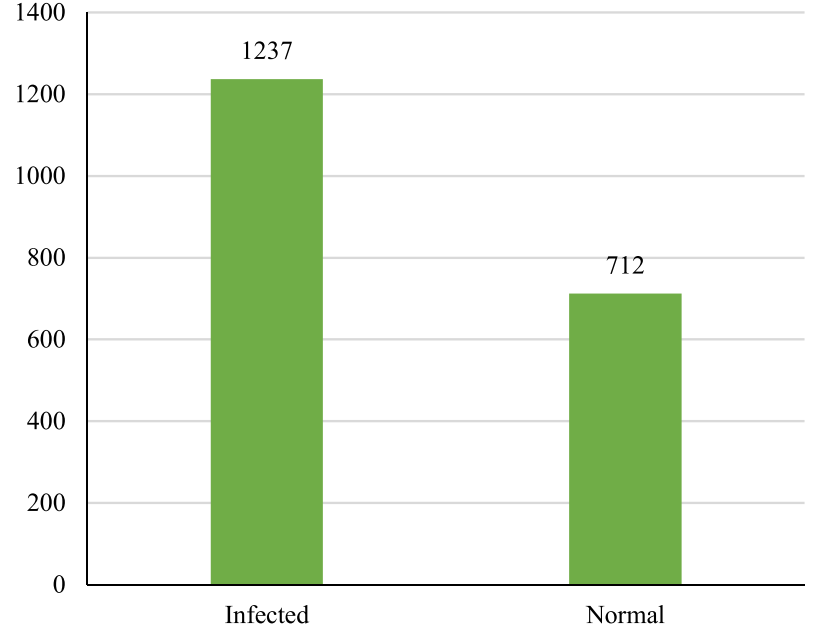

Fig. 3 Different classes of infected images

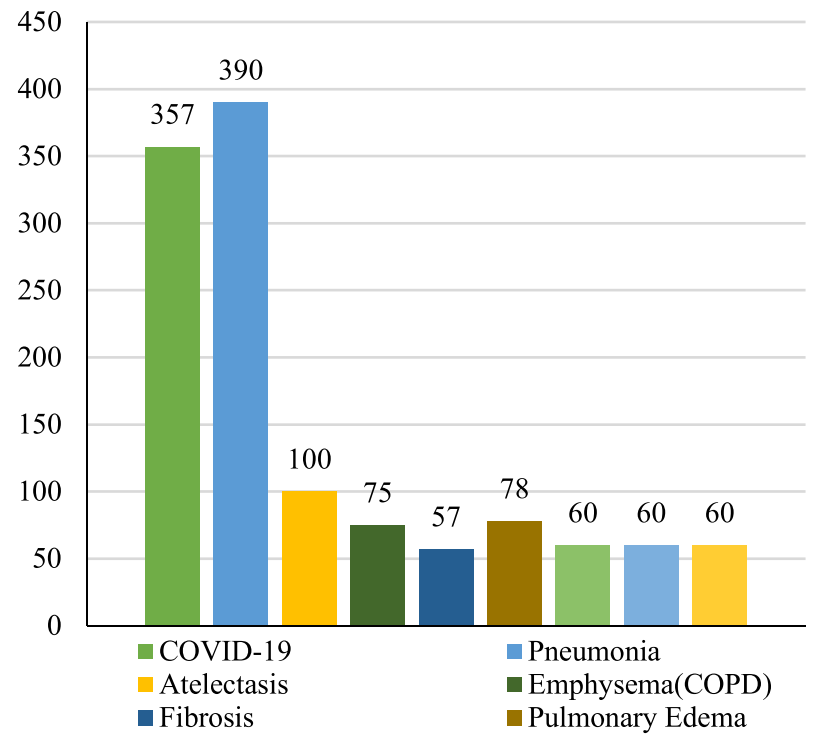

$225 \times 225$ in JPG/JPEG format, as shown in Fig. 4, for improving the performance of the projected DL network.

The 9 different classes of CPD infected images are COVID-19, Pneumonia, Atelectasis, Emphysema (COPD), Fibrosis, Pulmonary Edema, Pleural Thickening, Pneumothorax, and Nodule. The comparison among the CPD classes on basis of the average age of the infected patients is shown in Fig. 5. From the figure, it can be seen that 56 is the average age of COVID-infected patients. Figure 6 displays the COVID-19 death rates for every 1,000 persons by their sex and age in adults within the age group of 40 and above for different countries. It can be seen that the rate of male deaths is unfailingly greater than that of females in the age of 50 and above, irrespective of the mortality level [12]. For the purpose of evaluating the performance of model 2, the total infected images utilized for the proposed work are put into 3 major categories. These 3 major categories include 357 


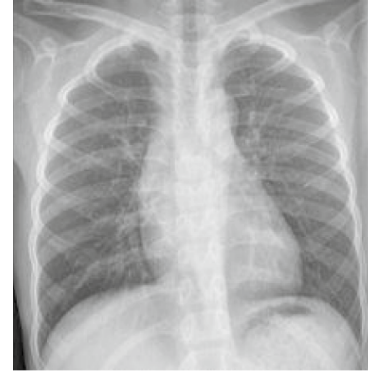

(a)

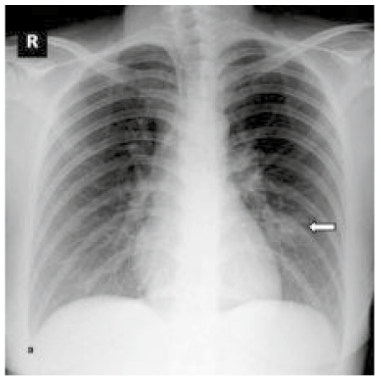

(b)

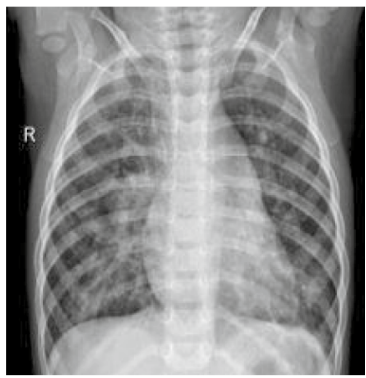

(c)

Fig. 4 Resized 225×225 (a) Normal (b) COVID-19 (c) Pneumonia infected CXR images

Fig. 5 Average age of CPD Infected Patients

Fig. 6 COVID-19 death rates by gender and age [12]
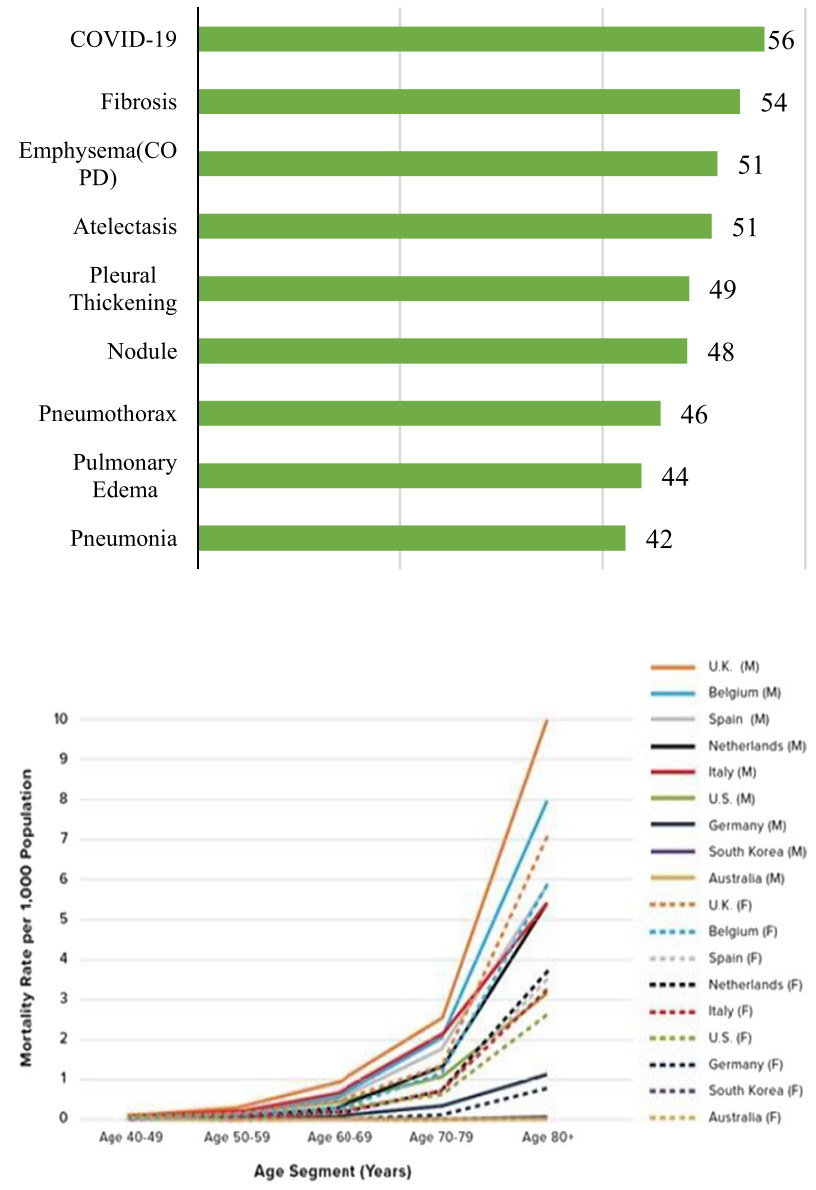
Fig. 7 Major Categories of CPD

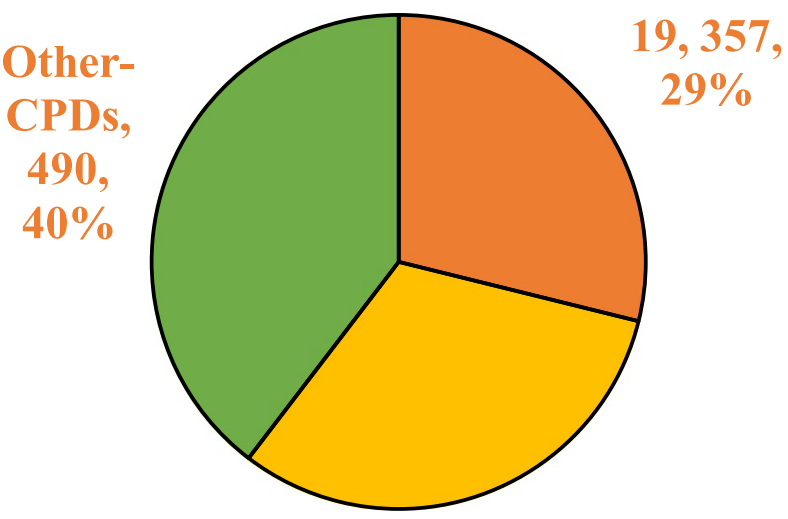

COVID infected images, 390 Pneumonia images, and 490 Chronic Pulmonary Disorder images, and the same is presented in Fig. 7.

\subsection{Pre-processing of images}

X-ray images of lungs are first passed through the pre-processing stage for the purpose of improving the image so as to make it more suitable for use. Primarily, the dimensions of the imageset are downscaled to $225 \times 225$ pixels from the original $1024 \times 1024$ as it supports the proposed DL framework to take significantly less processing time in giving the desired results. Median filtering is then employed for image quality enhancement as well as noise removal from the available X-ray images.

A non-linear median filtering operation is frequently utilized for image enhancement and to reduce the noise present in the image. It is quite effective in not only reducing the image noise but also in preserving the edges in the image as compared to convolution. In median filtering, each of the image pixels is filtered in sequence and whether the pixel represents its surroundings or not is decided by its neighbouring pixels. Also, in median filtering, the filter substitutes the pixel value with the median of the surrounding pixel values rather than replacing the pixel value with the mean value of neighbouring pixels. That is to say, that the pixel values from the surroundings are initially arranged in increasing order, and thereafter the said pixel value is substituted with the median value of the pixels. The median filtering operation is represented in Fig. 8.

$3 \mathrm{X} 3$ filter window

\begin{tabular}{|l|l|l}
\hline 30 & 20 & 10 \\
\hline 50 & 60 & 80 \\
\hline 40 & 70 & 90
\end{tabular}$\longrightarrow \rightarrow$\begin{tabular}{|c|c|c|}
\hline 10 & 20 & 30 \\
\hline 40 & 50 & 60 \\
\hline 70 & 80 & 90 \\
\hline
\end{tabular}$\longrightarrow$\begin{tabular}{|c|c|c|}
\hline- & - & - \\
\hline- & 50 & - \\
\hline- & - & - \\
\hline
\end{tabular}

Original Image Pixels values
Pixels arranged in ascending order
Output pixel value

Fig. 8 Median filter operation 
Extracting the desired region from an image is essential for improving the performance of the system by letting it focus only on the area of importance. To aid the DL network in extracting a pivotal feature from the image, we need to locate the region of interest (ROI) inside the images $[1,42,53]$. For the construction of ROI, it is significant to identify the possible regions of infection. In our proposed DL framework, we have focused only on the lung area in the image while removing the surrounding parts to form the ROI using a masked frame of fixed dimension. The masked image comprises all the input image pixels which lie entirely or moderately within the area surrounded by the frame. [18, 47]. Subsequently, the X-ray images are enhanced to make the region of interest more evident as displayed in Fig. 9 that shows the evaluation of the ROI extracted image against the enhanced X-ray image along with the correlation among them.

After the pre-processing stage and before distributing the imageset between the train and test data, the imageset is mixed up so as to make the proposed framework unbiased while working on the given data and avoid focusing on a small subset of the whole imageset. The available imageset is then parted into 3 sets namely training, testing, and validation. $70 \%$ of the total imageset is utilized for training the proposed framework while the remaining $30 \%$ data is equally divided and is utilized for testing as well as validation of the network.

\subsection{D-CNN based feature extraction}

Deep convolutional networks are a subclass of ANN having different layers and are primarily implemented to map multi-layered images for yielding the desired results after getting trained [31]. The key objective in employing the D-CNN is that it finds the pivotal features of an image by itself during the training process by engaging their convolutional filters. These convolutional filters are implemented in every layer of the network in order to develop feature tensors and stride defines the distance to which these filters will go. At large, when $\mathrm{s}_{1}$ is the length of the stride and $\mathrm{s}_{\mathrm{b}}$ the breadth of the stride, it can be expressed as:

$$
\left\lfloor\left(\eta_{l}-k_{l}+p_{r}+s_{l}\right) / s_{l}\right\rfloor \times\left\lfloor\left(\eta_{b}-k_{b}+p_{c}+s_{b}\right) / s_{b}\right\rfloor
$$

Where $n l \& n b$ are the length and breadth of the input tensor, $k l \& k b$ are the length and breadth of the kernel, $p r \& p c$ are the row and column of padding. For $p r=k l-1$ and $p c=k b-1$, the output will be given by:

$$
\left(\eta_{l}+s_{l}-1\right) / s_{l} \times\left(\eta_{b}+s_{b}-1\right) / s_{b}
$$

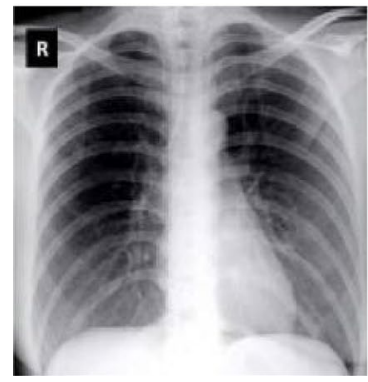

(a)

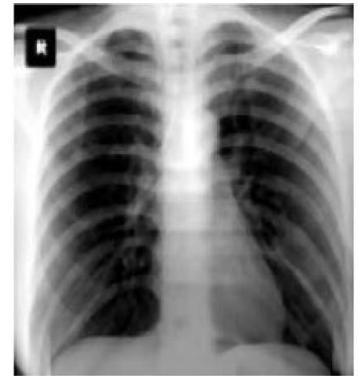

(b)

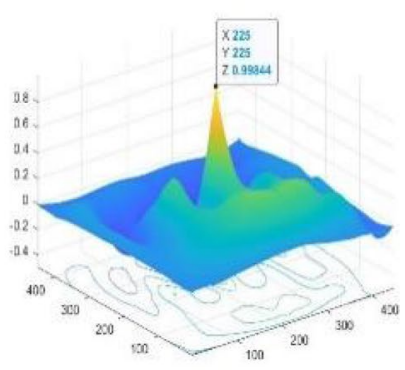

(c)

Fig. 9 (a) ROI of image (b) Enhanced ROI image (c) Correlation among (a) \& (b) 
Normally, a maximum stride of two pixels gives better results, and increasing the stride beyond this may result in the falloff of the output [26, 51]. Also, at the time of framing the stride, it is pivotal to see that the output should not be a fraction. Usually, zero paddings are needed if the convolutional filter doesn't cover all the available input image pixels as this is required to keep the spatial dimensions identical. The feature map tensors which are created utilizing the convolutional layer are expressed using Rectified Linear Unit (RLU) as the activation function. It is the most prevalent function, because of its upright performance and ease of execution. RLU gives an exceptionally straightforward nonlinear change. Assuming a component ' $x$ ', RLU is characterized as:

$$
R L U=\operatorname{Max}(x, 0)
$$

Normally, the activation function RLU holds only the positive value and rejects the negative values by putting the equivalent activation to 0 . The features obtained from the RLU are then given to the pooling layers for diminishing the dimensions. Maximum and Average pooling are the two recognized pooling functions, that are more frequently employed [30]. In order to standardize the feature map, a layer called batch normalization is usually employed next to the RLU layer. Such batch normalization layers are used for network adjustment as well as to fast-track the network training. An important point to be noted here is that while using the normalization layer, choosing the size of the batch might be considerably more noteworthy than without using it. Let $x \in B_{m}$ is input to batch normalization layer $B_{n}$ which belongs to the minibatch $B_{m}$, therefore the transform of $x$ is given by:

$$
B_{n}(x)=\vartheta \odot \frac{x-\sigma_{B_{m}}}{\mu_{B_{m}}}+\delta
$$

As choosing the unit variance is random, therefore an element-wise scaling as well as shifting parameters $\vartheta$ and $\delta$ having a similar profile is encompassed. It is important to be noted that $\vartheta$ and $\delta$ are required to be treated combinedly with various other parameters of the model. In the above expression $\sigma_{B_{m}}$ and $\mu_{B_{m}}$ denotes the mean and standard deviation of $B_{m}$ and is expressed as:

$$
\begin{gathered}
\sigma_{B_{m}}=\frac{1}{\left|B_{m}\right|} \sum_{x \in B_{m}} x \\
\mu_{B_{m}}=\frac{1}{\left|B_{m}\right|} \sum_{x \in B_{m}}\left(x-\sigma_{B_{m}}\right)^{2}+\theta
\end{gathered}
$$

It can be seen from the above equation that a constant $\theta>0$ is added to the variance to confirm that division by zero is never attempted, even when the empirical variance may have a chance to get vanished. The approximations of $\sigma_{B_{m}}$ and $\mu_{B_{m}}$ offsets the scaling issue through the use of noisy estimations of variance as well as mean. The concluding convolution layer is succeeded by a fully linked layer. The features that are resulted from the last convolutional layers also called pooling layers are then transformed into a vector of 1-dimension and are associated with a minimum of one densely connected layer to summarize the network's outcome [55]. Commonly the last layer of the deep network is the SoftMax layer that is employed for classifying the images and is expressed as:

$$
y_{k}=\frac{e^{k}}{\sum_{p=1}^{q} e^{q_{k}}}
$$


Finally, in order to classify the image, a classification layer is employed that works using cross-entropy to evaluate the misclassification loss and provides the ultimate estimation of the image labels. The loss approximation is expressed by:

$$
H(p, q)=-\sum_{f}(p(f) * \log (q(f)))
$$

Here ' $p$ ' specifies the image labels and $q(f)$ denotes the SoftMax layer output. In the proposed work finetuned DenseNet201 D-CNN is used for investigating and classifying CPDs using CXR images. The architecture of the proposed model is presented in Fig. 10.

\subsection{Transfer learning of pretrained densenet201}

Transfer learning (TL) is a technique in which the information obtained from a model that is trained on one dataset is used with an objective to forecast and classify labels pertaining to the dataset used in the other model [39]. The primary advantage of using the TL approach is that it reduced the time taken for the training of a particular model. Attuning a pre-trained system through TL is generally quite simpler as well as faster rather than starting the training from scratch. Employing pretrained networks permits the user to get trained into new tasks quickly and additionally, it also lowers the errors due to generalization. In this proposed work the information of a model that is trained for the classification of basic images is employed to explain the classification of chronic pulmonary disorders (CPD). Dense Convolutional Network-DenseNet201 is a pretrained DL network and as per its name, it has 201 densely connected layers. DenseNet is somewhat a logical augmentation of ResNet. Conventional CNN-like feedforward neural network links the $\mathrm{n}^{\text {th }}$ layer output to the input of $(n+1)^{\text {th }}$ layer [27], giving a layer of transition expressed by Eq. 8. ResNet, on the other hand, detours the non-linear transform by an expression given by Eq. 9 .

$$
\begin{gathered}
x_{n}=f_{n}\left(x_{n-1}\right) \\
x_{n}=f_{n}\left(x_{n-1}\right)+x_{n-1}
\end{gathered}
$$

A significant dissimilarity between DenseNet and ResNet is that in DenseNet there is a concatenation among the output of the layers instead of addition as in Eq. 10. Therefore, the $\mathrm{n}^{\text {th }}$ layer forms a chain of all the feature maps obtained as an input through the preceding layers.

$$
x_{n}=\left[x_{0}, f_{1}\left(x_{1}\right), f_{2}\left(\left[x_{0}, f_{1}\left(x_{1}\right)\right]\right), f_{3}\left(\left[x_{1}, f_{2}\left(x_{2}\right)\right]\right), . .\right]
$$

Here we have applied a TL approach along with finetuning on the DenseNet201 pretrained network for the classification of diseases using the CXR images. Firstly, the

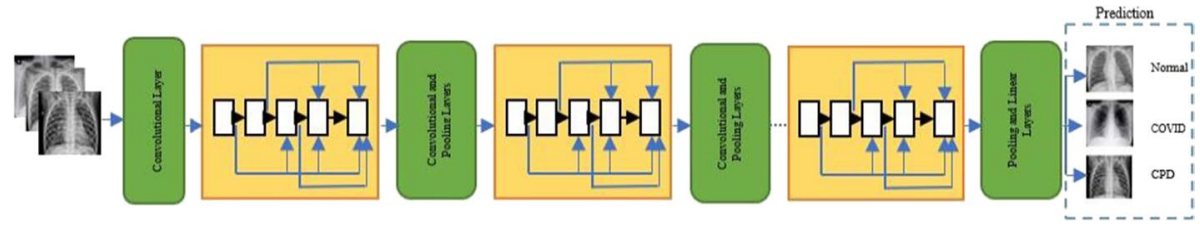

Fig. 10 The architecture of the proposed DenseNet201model 
DenseNet201 network is initialized and is set up for fine-tuning. The network is thus represented employing weights that are previously trained over ImageNet while the fully connected (FC) layer is left off. Starting from that point a new FC layer is designed that contains a Flatten layer, Dropout and Dense ("softmax") activation layer for the classification of CXR in both the models. Thereafter the weights of the convolutional layer of DenseNet201 are freeze so that the FC layer will only be trained for classification. The detailed layered structure of the proposed finetuned DenseNet201 model is presented in Fig. 11. Most of the D-CNN's utilize techniques for optimizing the objective function $f(x)$ by altering the value of $x$. Nevertheless, as this $f(x)$ is minimalized it becomes the cost (loss) function. Stochastic Gradient Descent is an optimization method which is used for optimizing $J(\theta)$, called the objective function, characterized by a constraint $\theta \in \mathbb{R}^{s}$ by reviewing it in the opposite course of $\nabla \theta J(\theta)$ relating to the parameters. The learning rate ' $\alpha$ ' represents the magnitude of the steps taken to attain the local minima. Stochastic Gradient Descent with Momentum is a method that quickens the descent and minimizes the oscillations in the path of descent by using $\gamma$ of the preceding step to the current vector update $[13,57]$. The algorithm employed for using the Stochastic Gradient Descent with Momentum is presented in algorithm 1.
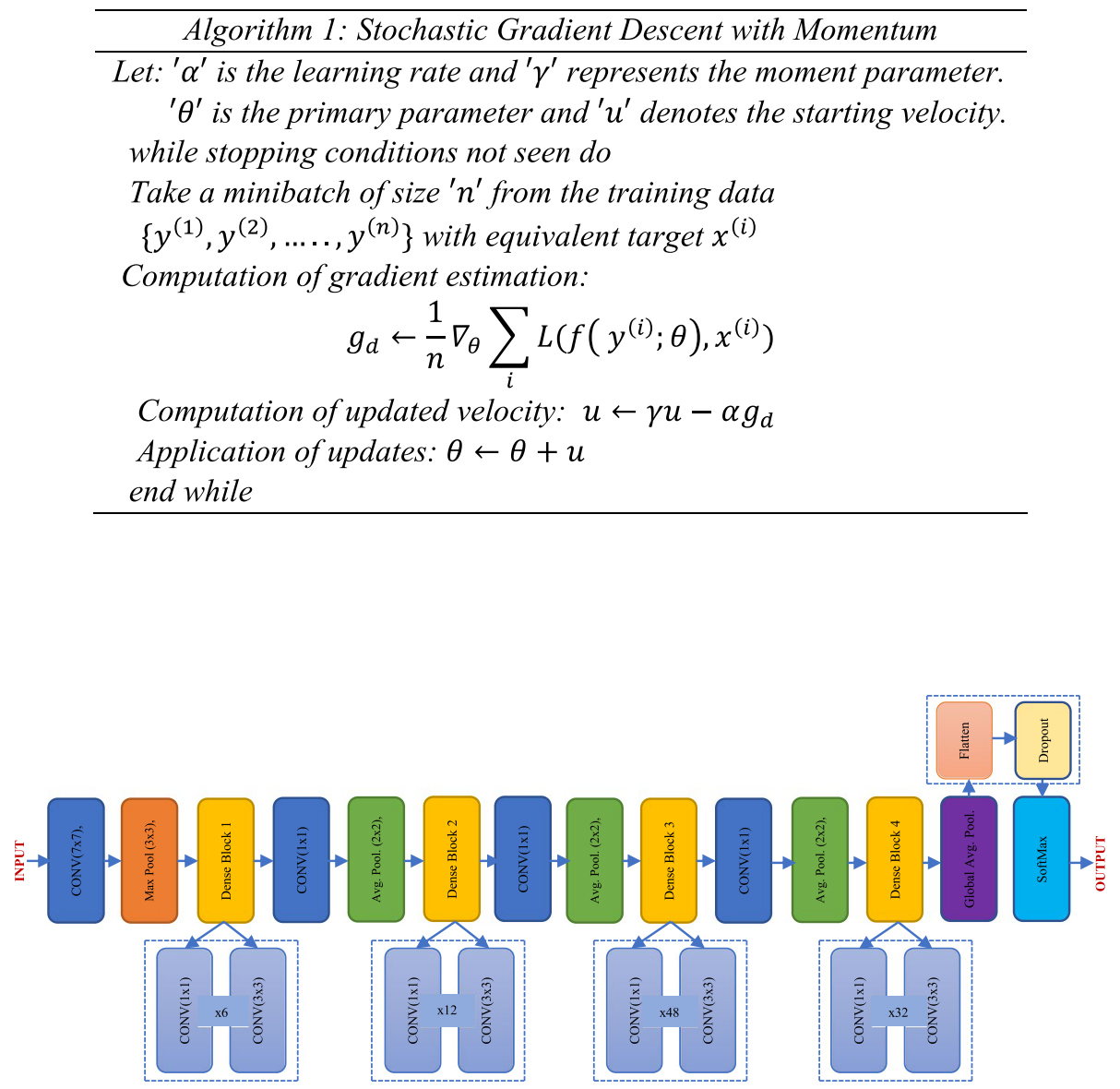

Fig. 11 Detailed layered structure of the proposed finetuned DenseNet201 model 


\subsection{Mathematical analysis}

Here the presented D-CNN-based classification models are evaluated against the other state-of-the-art models by making use of a variety of parameters obtained with the help of a confusion matrix. These parameters include sensitivity, specificity, precision, Cohen's kappa coefficient, F-score, error rate, and accuracy. The foremost aim of this research is twofold, firstly with the help of model 1, it will identify whether an individual is suffering from any pulmonary disorder or not, and secondly, with the help of model 2, it will classify the type of that pulmonary disorder. The results obtained from both models can be true or false. Also, the results for each individual might coordinate with the individual's real class. Therefore, here TP signifies the positive anticipated cases which are positive. TN connotes the foreseen negative cases which are actually negative. FN denotes the cases forecasted as negative but is positive in actuality. FP represents the cases estimated as positive but were negative in actuality. For performing the validating the proposed models following parameters are utilized.

(i) Sensitivity/Recall: It evaluates the capability of a particular model to make appropriate classification and is expressed as:

$$
\text { Sensitivity } / \text { Recall }=\frac{T P}{T P+F N}
$$

(ii) Specificity: It denotes the capability of a given system to exactly recognize the genuine $\mathrm{CPD}$ cases and is given as:

$$
\text { Specificity }=\frac{T N}{T N+F P}
$$

(iii) Precision: Closeness of two values is defined as precision and is expressed as:

$$
\text { Precision }=\frac{T P}{T P+F P}
$$

(iv) Accuracy: It defines the capability of a system to correctly identify the type of CPD cases. It is expressed as:

$$
\text { Accuracy }=\frac{T P+T N}{T P+T N+F P+F N}
$$

(v) Error Rate: The proportion of all the false estimations compared to the total dataset is called an error rate:

$$
\text { Error }=\frac{F P+F N}{T P+T N+F P+F N}
$$

(vi) F- Score: For the quantification of the achieved accuracy F-score is used. It is expressed as:

$$
F 1_{\text {score }}=\frac{2 *(\text { Precision } * \text { Recall })}{(\text { Precision }+ \text { Recall })}
$$

(vii) Cohen's kappa coefficient ( $\kappa)$ : It basically evaluates the inter-rater consistency for qualitative objects. It is expressed as: 


$$
\kappa=\frac{\text { Accuracy }- \text { RandomAccuracy }}{1-\text { RandomAccuracy }}
$$

Here Random Accuracy is calculated as:

RandomAccuracy $=P_{1} * P_{2}+\left(1-P_{1}\right) *\left(1-P_{2}\right)$

where:

$$
P_{1}=\frac{T P+F N}{T P+T N+F P+F N} \& P_{2}=\frac{T P+F P}{T P+T N+F P+F N}
$$

The values of the above-listed parameters must be appropriate for the best performance of the proposed models.

(viii) Geometric Mean (G-Mean): It evaluates the equilibrium among the performance of the classifier on the available classes. It is significant in the circumvention of underfitting and overfitting in the positive and negative classes respectively.

$$
G-\text { Mean }=\sqrt{\text { Recall } * \text { Specificity }}
$$

(ix) Youden's Index: It measures the classifier's capability of avoiding misclassifications. It gives identical weights on the performance of a classifier for both negative and positive cases:

$$
\text { Youden' sIndex }=\text { Recall }-(1-\text { Specificity })
$$

(x) Likelihood Ratio: It represents a way for assessing the performance of a classifier in terms of positive and negative likelihood ratios as:

$$
L R(+)=\frac{\text { Sensitivity }}{1-\text { Specificity }} ; L R(-)=\frac{1-\text { Sensitivity }}{\text { Specificity }}
$$

A higher value of LR (+) and a lower value of LR (-) is a sign of decent classifier performance.

(xi) Adjusted F-Score (AFS): This is an enhancement over the F-score particularly when there is an imbalance in the dataset and is computed as:

$$
A F S=\sqrt{F_{2} * F_{0.5}}
$$

where $\mathrm{F}_{2}=5 * \frac{\text { Recall } * \text { Precision }}{(4 * \text { Recall })+\text { Precesion }}$ andF $_{0.5}=\frac{5}{4} * \frac{\text { Recall } * \text { Precision }}{\left(0.5^{2} * \text { Recall }\right)+\text { Precesion }}$

(xii) Matthew's Correlation Coefficient (MCC): It is expressing the correlation coefficient between the observed and predicted class and is least affected by an imbalance in the dataset. It is expressed as:

$$
M C C=\frac{T P * T N-F P * F N}{\sqrt{(T P+F P)(T P+F N)(T N+F P)(T N+F N)}}
$$




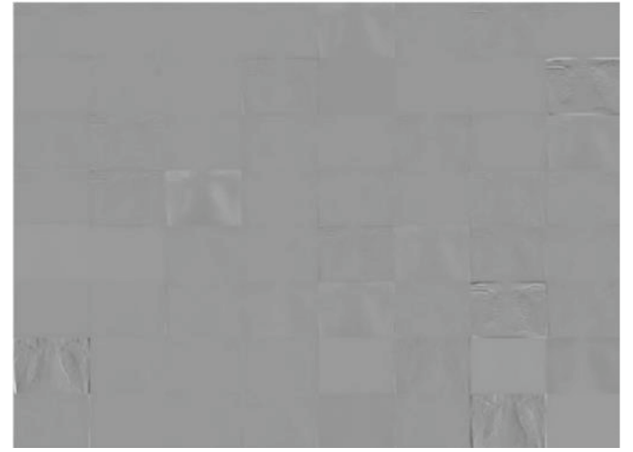

(a)

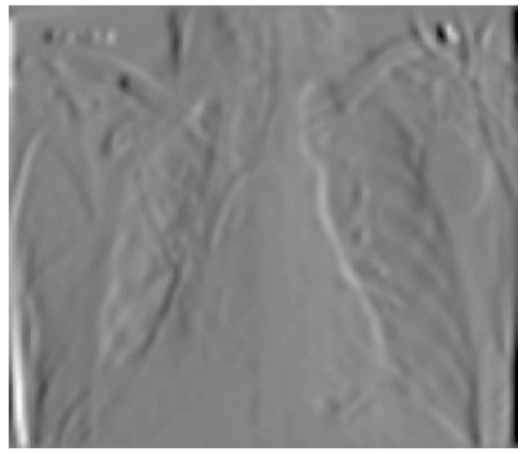

(b)

Fig. 12 (a) Activation of the 1st Conv. layer (b) Maximum activation within the 1st layer

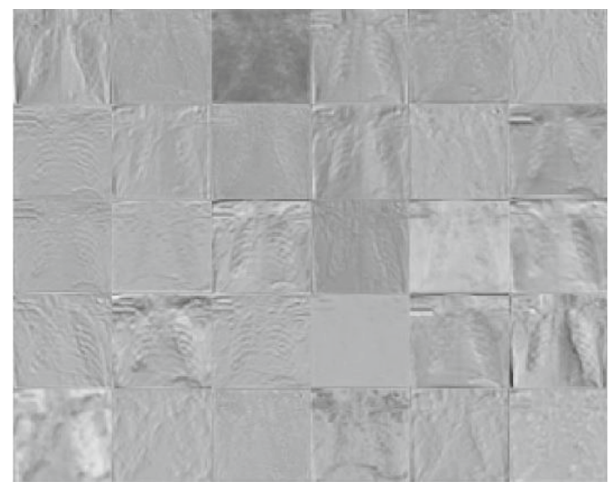

(a)

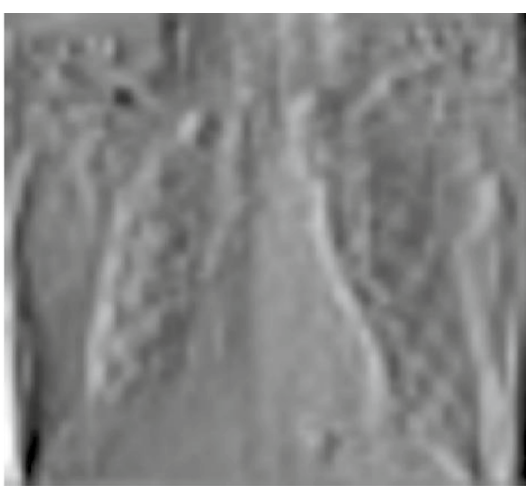

(b)

Fig. 13 (a) Activation of the 5th Conv. layer (b) Maximum activation within the 5th layer

\section{Analytical results and discussion}

A TL-based finetuned deep convolutional neural network (DenseNet201) is proposed here [23] for the precise identification of normal as well as chronic pulmonary disorders from CXR images. It also classifies with precision, the CPD images into pneumonia, COVID-19, and other CPD images. The two models discussed here are trained using DenseNet201 for achieving utmost accuracy. Here the identification of images is done through the Softmax layer of the finetuned D-CNN. Here an image is given as an input to the deep learning network and the activations in each layer are observed to determine the features learned by the network as compared to the input image. The initial layers of the D-CNN acquire basic image features like edges, corners, and colours, whereas more intricate features are learned at deeper layers. The activation of the first layer of the deep convolutional network DenseNet201 and the maximum activation within the first layer is shown in Fig. 12. Features of the layers at depth shape up by merging the features obtained from the previous layers. Figure 13 presents the investigation of the $5^{\text {th }}$ convolutional layer along with the maximum activation obtained in that layer. D-CNNs are more frequently regarded as an opaque system as there is no way of knowing the 
learning of a particular network or which input portion is accountable for the network prediction. When such CNNs fail in their predictions to give correct results, they fail enormously lacking any clarification. One of the methods by which we can get a visual description of the network predictions of D-CNNs is by Class activation mapping [52]. With the help of class activation mapping, we can easily check whether a particular portion of an image is making the network confused and directing it for making false predictions. Figure 14 represents the class activation maps for COVID and pneumoniainfected CXR images. The presented D-CNN DenseNet201 is trained using Stochastic gradient descent with momentum (SGDM) for both models to accomplish the best-inclass accuracy. Figure 15 presents the training accuracy as well as the loss for both models. A matrix of confusion represents how a given network performs classification on a given set of images for which the real labels are known already. Figure 16 shows such a matrix that displays the network performance of the two models in classifying CXR images. The horizontal axis gives the target class while the vertical axis represents the output in the confusion matrix. Table 1,2 and Table 3,4 show the derived values of sensitivity, specificity, precision, error rate, and accuracy along with the collective performance measurements for both the models. Performance Measurement of a classifier is a difficult task. Based on the type of applications, the complete rate of classification

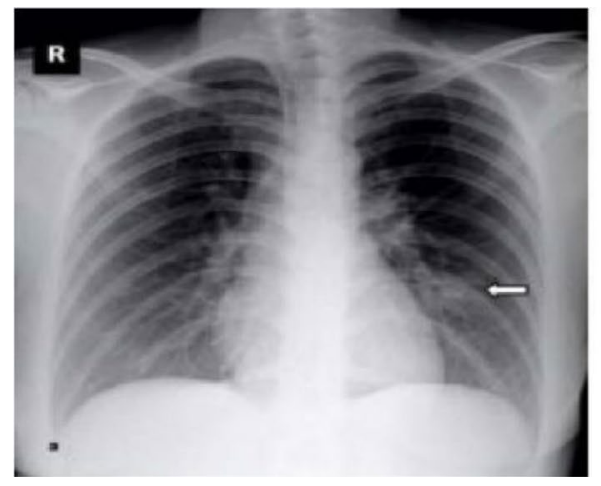

(a)

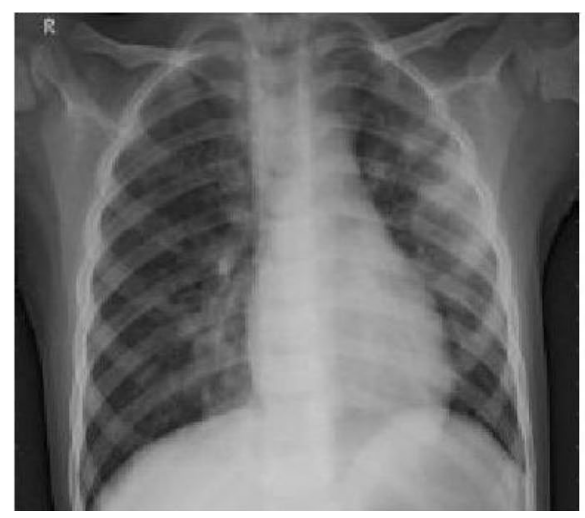

(c)

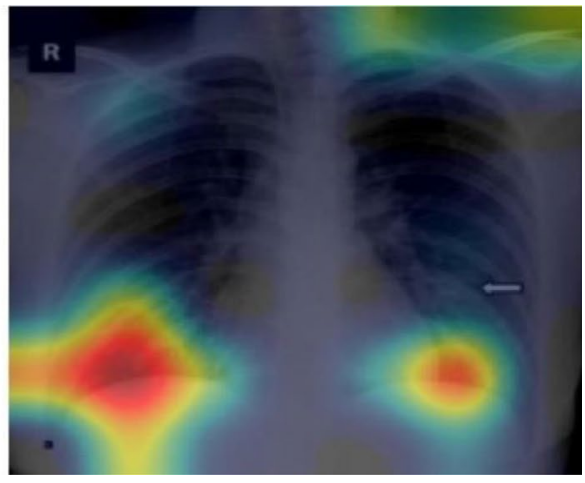

(b)

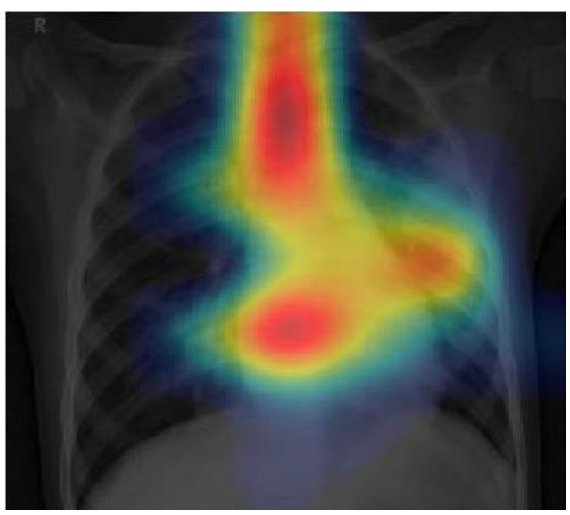

(d)

Fig. 14 Highlighted region for (a), (b) COVID and (c), (d) Pneumonia infected CXR images 

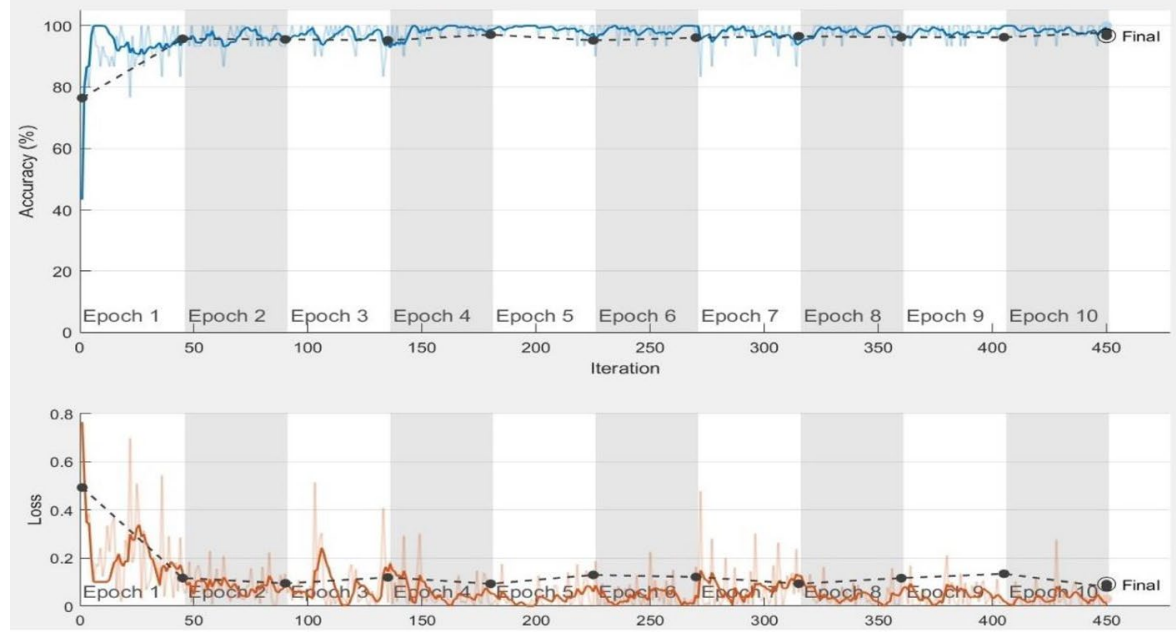

(a) Training accuracy of Model1: classification of Normal and Infected CXR Images

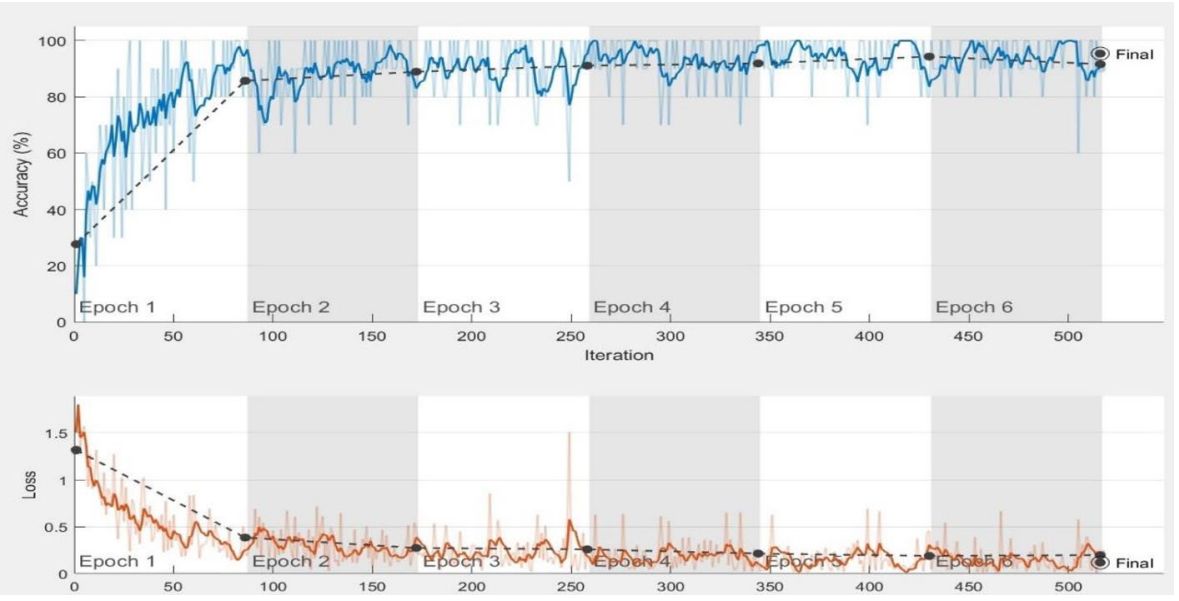

(b) Training accuracy of Model2: classification of COVID-19, Pneumonia and other CPD CXR Images

Fig. 15 Training accuracy and loss of a) Model 1, b) Model 2

may vary if one or more predictions fail. Kappa coefficient basically evaluates the interrater consistency for qualitative objects. Also, the Adjusted F-score value is an enhancement over the F-score particularly when there is an imbalance in the dataset. We have also taken into account the Geometric mean (G-Mean), Correlation Coefficient (MCC), Youden's index, and Likelihood parameters for estimating the collective performance of the system in classifying different classes by abstaining from misclassification and assessing the classification accuracy. These measures are usually considered as a much efficient way of measuring the accuracy of a prediction model as it also considers the occurrence of an agreement by chance. From the observation given in Table 2, it is clear that the presented model 1 has achieved the Kappa coefficient, G-Mean, Youden's Index, and MCC values of $93.08 \%, 97.13 \%, 94.28 \%$, and $93.19 \%$ respectively in the prediction of both infected as well as normal images whereas the F-score and Adjusted 


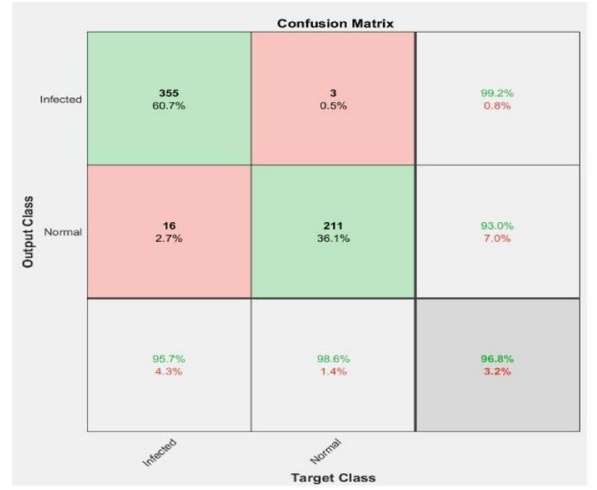

(a)

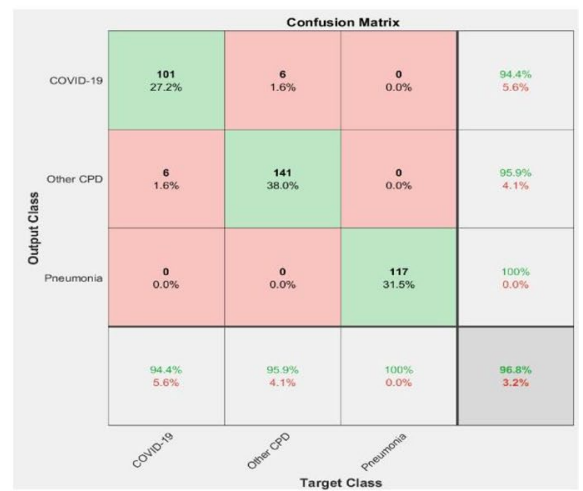

(b)

Fig. 16 (a) Confusion matrix of model 1 (b) Confusion matrix of model 2

Table 1 Analysis of Confusion matrix for Model 1

\begin{tabular}{llllllllll}
\hline Types & TP & TN & FP & FN & Recall & Specificity & Precision & Error rate & Accuracy \\
\hline Infected & 355 & 211 & 3 & 16 & 0.9568 & 0.9859 & 0.9916 & 0.0324 & 0.9675 \\
Normal & 211 & 355 & 16 & 3 & 0.9859 & 0.9568 & 0.9295 & 0.0324 & 0.9675 \\
Overall Accuracy & $\mathbf{9 6 . 8 \%}$ & & & & & & & \\
\hline
\end{tabular}

Table 2 Collective Performance Measurements for Model 1

\begin{tabular}{llllllll}
\hline Types & G-Mean & $\begin{array}{l}\text { Cohen's } \\
\text { kappa coeff. } \\
(\kappa)\end{array}$ & F1 Score & Youden's Index & Likelihood Ratio & AFS & MCC \\
\hline Infected & 0.9713 & 0.9308 & 0.9739 & 0.9428 & $\begin{array}{l}\text { LR (+) } 68.2569 \\
\text { LR (-) } 0.0437\end{array}$ & 0.9739 & 0.9319 \\
Normal & 0.9713 & 0.9308 & 0.9569 & 0.9428 & $\begin{array}{l}\text { LR (+) } 22.8624 \\
\text { LR (-) } 0.0146\end{array}$ & 0.9570 & 0.9319 \\
& & & & & & &
\end{tabular}

Table 3 Analysis of Confusion matrix for Model 2

\begin{tabular}{llllllllll}
\hline Types & TP & TN & FP & FN & Recall & Specificity & Precision & Error rate & Accuracy \\
\hline COVID-19 & 101 & 258 & 6 & 6 & 0.9439 & 0.9772 & 0.9439 & 0.0323 & 0.9676 \\
Other CPD & 141 & 218 & 6 & 6 & 0.9591 & 0.9732 & 0.9591 & 0.0323 & 0.9676 \\
Pneumonia & 117 & 254 & 0 & 0 & 1 & 1 & 1 & 0 & 1 \\
Overall Accuracy & $\mathbf{9 6 . 8 \%}$ & & & & & & & \\
\hline
\end{tabular}

F-score values are $97.39 \%$ for infected and $95.69 \%$ for normal images. Whereas in Table 2 the Kappa coefficient, G-Mean, Youden's Index and MCC values for COVID classification are $92.1 \%, 96.04 \%, 92.11 \%$, and $92.11 \%$ respectively for Other CPD 
Table 4 Collective Performance Measurements for Model 2

\begin{tabular}{|c|c|c|c|c|c|c|c|}
\hline Types & G-Mean & $\begin{array}{l}\text { Cohen's } \\
\text { kappa coeff. } \\
\text { (к) }\end{array}$ & F1 Score & Youden's Index & Likelihood Ratio & AFS & $\mathrm{MCC}$ \\
\hline COVID-19 & 0.9604 & 0.9210 & 0.9439 & 0.9211 & $\begin{array}{l}\mathrm{LR}(+) 41.39 \\
\mathrm{LR}(-) 0.057\end{array}$ & 0.9439 & 0.9211 \\
\hline Other CPD & 0.9661 & 0.9322 & 0.9591 & 0.9323 & $\begin{array}{l}\mathrm{LR}(+) 35.78 \\
\mathrm{LR}(-) 0.042\end{array}$ & 0.9591 & 0.9323 \\
\hline Pneumonia & 1 & 1 & 1 & 1 & $\begin{array}{l}\operatorname{LR}(+) \infty \\
\operatorname{LR}(-) 0\end{array}$ & 1 & 1 \\
\hline
\end{tabular}

classification Kappa coefficient, G-Mean is 93.22\%, 96.61\%, and Youden's Index and MCC is $93.23 \%$ respectively while for classifying pneumonia it is $100 \%$ which shows that the classification models presented here are quite robust and efficient. Also, the F1 Score is an improved measure to employ if an equilibrium is required amid Recall and Precision and if there exists an irregular distribution of classes. In the tables above the F score for both the models have achieved quite a good value. From the data presented in Table 1, the F-score for infected and normal images is $97.39 \%$ and $95.69 \%$ respectively. While for model 2 the F-score for COVID, Other CPDs, and pneumonia infected images are $94.39 \%, 95.91 \%$, and $100 \%$ respectively.

Area Under Curve (AUC) and Receiver Operating Characteristics (ROC) is a classification measurement for problems at several thresholds. It is basically a curve representing the probability and the degree of separability. It explains how proficient the model is in classifying different classes. Higher is the value of AUC, better is the prediction of the model in categorizing the infected and normal CXR images. The ROC curve for both models is shown in Fig. 17. G-Mean and Youden's Index Adjusted F score and MCC which are employed to measure the classifier performance are also giving promising results for both the presented models.

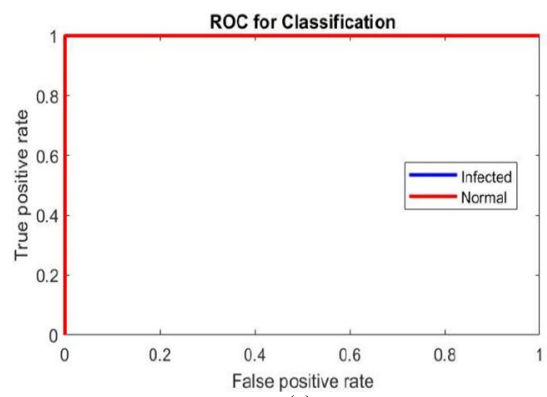

(a)

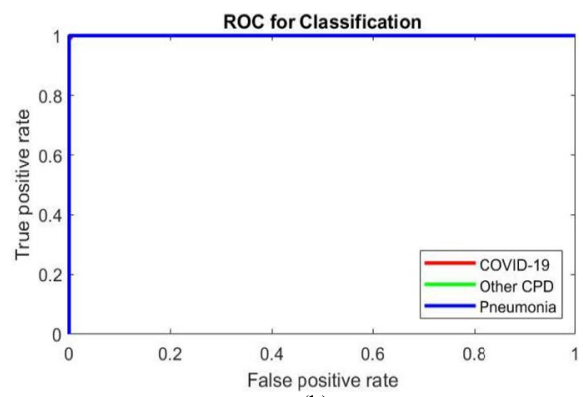

(b)

Fig. 17 (a) ROC for classification model 1 (b) ROC for classification model 2 


\section{Comparative analysis}

The presented research work provides a framework for identifying the CXR images and classifying them into normal and infected images in model 1 and COVID, Other Chronic Pulmonary Disorder (OCPD), and pneumonia infected images in model 2. The presented models accomplish the extraction of features by themselves as per the architecture of the DL-CNN. The final layers of the proposed DenseNet201 network are finetuned for the classification of the new task. The CXR images are resized to $225 \times 225$ in JPG/JPEG. MATLAB R2019a is employed on an i5 Nvidia-enabled $10^{\text {th }}$ generation processor for the execution of the architecture proposed. Table 5 presents a comparative analysis of several associated State of the Art (SoA) works.

\section{Conclusion}

Infection in the lungs poses respirational complications with various indications like breathing difficulty, cough, high fever, and pneumonia. WHO had identified cancer in the lungs as a fatal cancer type amongst others and thus, the timely detection of such cancer is pivotal for an individual's health. Numerous deep learning-based image processing frameworks which include visual geometry group-based neural network (VGG), residual neural network (ResNet), convolutional neural network (CNN), etc. are utilized for predicting lung diseases. Since the elementary convolutional neural networks have performed poorly in identifying the atypical image, in the presented research work, a novel D-CNN based on the TL approach is investigated for the identification of chronic pulmonary disorders using DenseNet201 architecture. Here two classification models are proposed. Model 1 aims to identify whether a given CXR image is a normal image or an infected one. Whereas model 2 further investigates the type of CPD with which the CXR image is infected if found so by model 1. Model 2 categorizes the infected CXR images into 3 major categories i.e.,

Table 5 Comparative analysis of associated State of the Art (SoA) work

\begin{tabular}{|c|c|c|c|c|c|c|}
\hline Methodology & Modality & Normal Images & COVID-19 & Other CPD & Total Images & $\begin{array}{l}\text { Clas- } \\
\text { sification } \\
\text { Accuracy }\end{array}$ \\
\hline $\begin{array}{l}\text { Wang \& Wong,2020 } \\
\text { [53] }\end{array}$ & CXR & 8066 & 53 & 5526 & 13,645 & $92 \%$ \\
\hline Hemdan et al.,2020 [18] & CXR & 25 & 25 & NA & 50 & $90 \%$ \\
\hline Song Y. et al.,2020 [47] & $\mathrm{CT}$ & 708 & 777 & NA & 1485 & $86 \%$ \\
\hline Sethy P. et al.,2020 [42] & CXR & 25 & 25 & NA & 50 & $95 \%$ \\
\hline $\begin{array}{l}\text { Apostolopoulos } \\
\text { et al.,2020 [1] }\end{array}$ & CXR & 504 & 224 & 700 & 1428 & $93 \%$ \\
\hline $\begin{array}{l}\text { Khan, Shah and Bhat, } \\
2020 \text { [31] }\end{array}$ & CXR & 310 & 284 & 657 & 1251 & $89.6 \%$ \\
\hline Jaiswal et al., 2020 [23] & $\mathrm{CT}$ & 1230 & 1262 & NA & 2492 & $96 \%$ \\
\hline Proposed Work & CXR & 712 & 357 & 880 & 1949 & $\begin{array}{l}\text { Model 1: } \\
\text { 96.8\% } \\
\text { Model } \\
\text { 2: } \\
96.8 \%\end{array}$ \\
\hline
\end{tabular}


COVID-19, Pneumonia, and Other CPD infected images. Additionally, the infected areas identified by the proposed model are highlighted to offer explainability of detection. As presented by the thorough analysis of the observational results and utilizing an imageset of 1949 CXR images, model1 obtained an overall accuracy of $96.8 \%$ whereas model 2 gives an accuracy of $96.8 \%$ in categorizing the infected images into 3 major categories. Such an automated system may help the radiologist in substantiating their diagnosis and in starting well in time treatment of these deadly lung diseases. Apart from the results discussed, this work suffers from few limitations one of which is the unavailability of a sufficient dataset pertaining to different classes like Fibrosis, Atelectasis, Emphysema, etc. because of which in this work these mentioned infected classes are combined into one and named as other chronic pulmonary disorders. Also, this work focuses only on the X-ray images of the chest which makes the useability of this proposed system somewhat restricted. In the future, we will try to work on multiple image modalities and investigate the presented research work on an extensive imageset of CPD for multiple disease classes. Additionally, we will try to enhance the performance using feature extraction methods that are more sophisticated.

\section{References}

1. Apostolopoulos ID, Mpesiana TA (2020) Covid-19: automatic detection from X-ray images utilizing transfer learning with convolutional neural networks. Phys Eng Sci Med 1

2. Baker JA, Rosen EL, Lo JY, Gimenez EI, Walsh R, Soo MS (2003) Computer-aided detection (CAD) in screening mammography: sensitivity of commercial CAD systems for detecting architectural distortion. Am J Roentgenol 181(4):1083-1088

3. Barrientos F, Roman-Gonzalez A, Barrientos R, Solis L, Alva A, Correa M, ..., Oberhelman R (2016) Filtering of the skin portion on lung ultrasound digital images to facilitate automatic diagnostics of pneumonia. In 2016 IEEE 36th Central American and Panama Convention (CONCAPAN XXXVI) (pp. 1-4). IEEE

4. Barrientos R, Roman-Gonzalez A, Barrientos F, Solis L, Correa M, Pajuelo M, ..., Checkley W (2016) Automatic detection of pneumonia analyzing ultrasound digital images. In 2016 IEEE 36th Central American and Panama Convention (CONCAPAN XXXVI) (pp. 1-4). IEEE

5. Behzadi-khormouji $\mathrm{H}$ et al (2019) Deep learning, reusable and problem based architectures for detection of consolidation on chest X-ray images. Comput Methods Progr Biomed. https://doi.org/10.1016/j. cmpb.2019.105162

6. Bhandary Abhir et al (2020) Deep-learning framework to detect lung abnormality - a study with chest X-Ray and lung CT scan images. Pattern Recogn Lett 129:271-8. https://doi.org/10.1016/j.patrec. 2019.11.013

7. Bharati S, Podder P, Paul PK (2019) Lung cancer recognition and prediction according to random forest ensemble and RUSBoost algorithm using LIDC data. Int J Hybrid Intell Syst 15(2):91-100. https:// doi.org/10.3233/HIS-190263

8. Cheng JZ, Ni D, Chou YH, Qin J, Tiu CM, Chang YC, ..., Chen CM (2016) Computer-aided diagnosis with deep learning architecture: applications to breast lesions in US images and pulmonary nodules in CT scans. Sci Rep 6(1): 1-13

9. Cheng YT, Lin YF, Chiang KH, Tseng VS (2017) Mining sequential risk patterns from large-scale clinical databases for early assessment of chronic diseases: a case study on chronic obstructive pulmonary disease. IEEE J Biomed Health Inf 21:303-311

10. Chouhan V et al (2020) A novel transfer learning based approach for pneumonia detection in chest X-ray images. Appl Sci 10(2):559. https://doi.org/10.3390/app10020559

11. Cisneros-Velarde P, Correa M, Mayta H, Anticona C, Pajuelo M, Oberhelman R, ..., Lavarello R (2016) Automatic pneumonia detection based on ultrasound video analysis. In 2016 38th Annual International Conference of the IEEE Engineering in Medicine and Biology Society (EMBC) (pp. 41174120). IEEE

12. COVID-19 Mortality Rates by Age and Gender: Why Is the Disease Killing More Men than Women? (n.d.). Retrieved December 3, 2020, from https://www.rgare.com/knowledge-center/media/research/ covid-19-mortality-rates-by-age-and-gender-why-is-the-disease-killing-more-men-than-women 
13. Datta P, Gupta A, Agrawal R (2014) Statistical modeling of B-Mode clinical kidney images. In 2014 International Conference on Medical Imaging, m-Health and Emerging Communication Systems (MedCom) (pp. 222-229). IEEE

14. Doi K (2007) Computer-aided diagnosis in medical imaging: historical review, current status and future potential. Comput Med Imaging Graph 31(4-5):198-211

15. Eshaghi H, Ziaee V, Khodabande M, Safavi M, Haji Esmaeil Memar E (2021) Clinical Misdiagnosis of COVID-19 Infection with Confusing Clinical Course. Case Rep Infect Dis 2021https://doi. org/10.1155/2021/6629966

16. Gu Y, Lu X, Yang L, Zhang B, Yu D, Zhao Y, Gao L, Wu L, Zhou T (2018) Automatic lung nodule detection using a 3D deep convolutional neural network combined with a multi-scale prediction strategy in chest CTs. Comput Biol Med 103:220-231

17. He K, Gkioxari G, Dollar P, Girshick R. Mask r-cnn. Proceedings of the IEEE International Conference on Computer Vision 2017:2961-9

18. Hemdan EED, Shouman MA, Karar ME (2020) Covidx-net: A framework of deep learning classifiers to diagnose covid-19 in X-ray images. arXiv preprint arXiv:2003.11055

19. Hina K, Khalid S, Akbar MU (2016) A review on automatic tuberculosis screening using chest radiographs. In 2016 Sixth International Conference on Innovative Computing Technology (INTECH) (pp. 285-289). IEEE

20. Horváth G, Orbán G, Horváth Á, Simkó G, Pataki B, Máday P, Juhász S (2009) A cad system for screening X-ray chest radiography. In World Congress on Medical Physics and Biomedical Engineering, September 7-12, 2009, Munich, Germany (pp. 210-213). Springer, Berlin, Heidelberg

21. Huang J, Rathod V, Sun C, Zhu M, Korattikara A, Fathi A, Fischer I, Wojna Z, Song Y, Guadarrama $S$, et al. Speed/accuracy trade-offs for modern convolutional object detectors. Proceedings of the IEEE Conference on Computer Vision and Pattern Recognition 2017:7310-1

22. Irvin J, Rajpurkar P, Ko M, Yu Y, Ciurea-Ilcus S, Chute C, Marklund H, Haghgoo B, Ball r, Shpanskaya K, et al. Chexpert: a large chest radiograph dataset with uncertainty labels and expert comparison. 2019. arXiv:1901.07031

23. Jaiswal A, Gianchandani N, Singh D, Kumar V, Kaur M (2020) Classification of the COVID-19 infected patients using DenseNet201 based deep transfer learning. J Biomol Struct Dyn 1-8. https:// doi.org/10.1080/07391102.2020.1788642

24. Kallianos K, Mongan J, Antani S et al (2019) How far have we come? Artificial intelligence for chest radiograph interpretation. Clin Radiol 74(5):338-345. https://doi.org/10.1016/j.crad.2018.12. 015

25. Karargyris A, Antani S, Thoma G (2011) Segmenting anatomy in chest X-rays for tuberculosis screening. In 2011 Annual International Conference of the IEEE Engineering in Medicine and Biology Society (pp. 7779-7782). IEEE

26. Khan AI, Shah JL, Bhat MM (2020) CoroNet: A deep neural network for detection and diagnosis of COVID-19 from chest X-ray images. Comput Methods Programs Biomed 196:105581. https://doi. org/10.1016/j.cmpb.2020.105581

27. Krizhevsky A, Sutskever I, Hinton GE (2017) Imagenet classification with deep convolutional neural networks. Commun ACM 60(6):84-90

28. Kuan K, Ravaut M, Manek G, Chen H, Lin J, Nazir B, Chen C, Howe TC, Zeng Z, Chandrasekhar V (2017) Deep learning for lung cancer detection: tackling the Kaggle data science bowl 2017 challenge. https://arxiv.org/abs/1705.09435. Accessed 12 Dec 2020

29. Liang C-H et al (2019) Identifying pulmonary nodules or masses on chest radiography using deep learning: external validation and strategies to improve clinical practice. Clin Radiol. https://doi.org/ 10.1016/j.crad.2019.08.005

30. Liu J, Pan Y, Li M, Chen Z, Tang L, Lu C, Wang J (2018) Applications of deep learning to MRI images: A survey. Big Data Mining and Analytics 1(1):1-18

31. Lundervold AS, Lundervold A (2019) An overview of deep learning in medical imaging focusing on MRI. Z Med Phys 29(2):102-127

32. Mehrotra R, Ansari MA, Agrawal R, Anand RS (2020) A Transfer Learning approach for AI-based classification of brain tumors. Mach Learn Appl 2:100003

33. Mohammed MA, Abdulkareem KH, Garcia-Zapirain B, Mostafa SA, Maashi MS, Al-Waisy AS, ..., Le DN (2020) A comprehensive investigation of machine learning feature extraction and classification methods for automated diagnosis of covid-19 based on x-ray images. Comput Mater Continua 66(3)

34. Mondal MRH, Bharati S, Podder P, Podder P. "Data analytics for novel coronavirus disease", informatics in medicine unlocked, 20. Elsevier; 2020. p. 100374. https://doi.org/10.1016/j.imu.2020.100374

35. Murray CJL, Lopez AD (1997) Alternative projections of mortality and disability by cause 19902020: global burden of disease study. Lancet 349:1498-1504 
36. Nasrullah N, Sang J, Alam MS, Xiang H. Automated detection and classification for early stage lung cancer on CT images using deep learning. Proc SPIE 13 May 2019; 10995:109950S. Pattern Recognition and Tracking XXX

37. Nielsen KG, Bisgaard H (2005) The effect of inhaled budesonide on symptoms, lung function, and cold air and methacholine responsiveness in 2- to 5-year-old asthmatic children. Am J Respir Crit Care Med 162:1500-1506

38. NIH sample Chest X-rays dataset. https://www.kaggle.com/nih-chest-xrays/sa mple. [Accessed 28 June 2020]

39. Pan SJ, Yang Q (2009) A survey on transfer learning. IEEE Trans Knowl Data Eng 22(10):1345-1359

40. Ren S, He K, Girshick R, Sun J. Faster r-cnn: towards real-time object detection with region proposal networks. Adv Neural Inf Process Syst 2015:91-9. 6th ACM conference on bioinformatics, computational biology and health informatics. Atlanta, GA, USA: ACM; 2015. p. 258-67

41. Ronneberger O, Fischer P, Brox T (2015) U-net: convolutional networks for biomedical image segmentation. In: International conference on medical image computing and computer-assisted intervention, vol. 9351. Berlin/Heidelberg, Germany: Springer p. 234-41

42. Sethy PK, Behera SK (2020) Detection of coronavirus disease (covid-19) based on deep features. Preprints 2020030300:2020

43. Setio AAA, Traverso A, de Bel T, Berens MSN, van den Bogaard C, Cerello P, Chen H, Dou Q, Fantacci ME, Geurts B et al (2017) Validation, comparison, and combination of algorithms for automatic detection of pulmonary nodules in computed tomography images: the LUNA16 challenge. Med Image Anal 42:1-13

44. Shelhamer E, Long J, Darrell T (2017) Fully convolutional networks for semantic segmentation. IEEE Trans Pattern Anal Mach Intell 39(4):640-651. https://doi.org/10.1109/TPAMI.2016.2572683

45. Shrivastava A, Sukthankar R, Malik J, Gupta A. Beyond skip connections: topdown modulation for object detection. 2017.arXiv:1612.06851

46. Song Q, Zhao L, Luo X, Dou X (2017) Using deep learning for classification of lung nodules on computed tomography images. J Healthc Eng 8314740. https://doi.org/10.1155/2017/8314740

47. Song Y, Zheng S, Li L, Zhang X, Zhang X, Huang Z, ..., Chong Y (2020) Deep learning enables accurate diagnosis of novel coronavirus (COVID-19) with CT images. medRxiv

48. Sun W, Zheng B, Qian W (2016) Computer aided lung cancer diagnosis with deep learning algorithms. In: Proc SPIE. Medical Imaging, 2016, 9785. Computer-Aided Diagnosis 97850Z. https://doi.org/10. $1117 / 12.2216307$

49. Sun W, Zheng B, Qian W (2017) Automatic feature learning using multichannel ROI based on deep structured algorithms for computerized lung cancer diagnosis. Comput Biol Med 89:530-539

50. Tuberculosis Chest X-ray Image Data Sets. - LHNCBC Abstract. Available at: https://lhncbc.nlm.nih. gov/LHC-publications/pubs/TuberculosisChestXrayImageDataSets.html (Accessed: 16 August 2021)

51. Van der Burgh HK, Schmidt R, Westeneng HJ, de Reus MA, van den Berg LH, van den Heuvel MP (2017) Deep learning predictions of survival based on MRI in amyotrophic lateral sclerosis. NeuroImage: Clinical 13:361-369

52. Vieira SM, Kaymak U, Sousa JM (2010) Cohen's kappa coefficient as a performance measure for feature selection. In International Conference on Fuzzy Systems (pp. 1-8). IEEE

53. Wang L, Wong A (2020) COVID-Net: A Tailored Deep Convolutional Neural Network Design for Detection of COVID-19 Cases from Chest X-Ray Images. arXiv preprint arXiv:2003.09871

54. World Lung Day 2019: Healthy Lungs for All - Global Initiative for Chronic Obstructive Lung Disease - GOLD, https://goldcopd.org/world-lung-day-2019-healthy-lungs-for-all/. Accessed 27 Nov 2020

55. Yamashita R, Nishio M, Do RKG, Togashi K (2018) Convolutional neural networks: an overview and application in radiology. Insights Imaging 9(4):611-629

56. Zenteno O, Castañeda B, Lavarello R (2016) Spectral-based pneumonia detection tool using ultrasound data from pediatric populations. In 2016 38th Annual International Conference of the IEEE Engineering in Medicine and Biology Society (EMBC) (pp. 4129-4132). IEEE

57. Zhou, Bolei, Aditya Khosla, Agata Lapedriza, Aude Oliva, and Antonio Torralba. "Learning deep features for discriminative localization." In Proceedings of the IEEE Conference on Computer Vision and Pattern Recognition, pp. 2921-2929. 2016

58. Zhu W, Liu C, Fan W, Xie X (2018) DeepLung. Deep 3D dual path nets for automated pulmonary nodule detection and classification. In: Proceedings of the IEEE winter conference on applications of computer vision (WACV); 12-15 March. p. 673-81. Lake Tahoe, NV, USA

Publisher's Note Springer Nature remains neutral with regard to jurisdictional claims in published maps and institutional affiliations. 


\section{Authors and Affiliations}

\section{Rajat Mehrotra $^{1}$ (D) Rajeev Agrawal $^{2}$ - M. A. Ansari ${ }^{3}$}

Rajeev Agrawal

rajkecd@gmail.com

M. A. Ansari

ma.ansari@gbu.ac.in

1 Department of Electrical \& Electronics Engineering, GL Bajaj Institute of Technology \& Management, Gr. Noida, India

2 Department of Electronics \& Communication Engineering, GL Bajaj Institute of Technology \& Management, Gr. Noida, India

3 Department of Electrical Engineering, School of Engineering, Gautam Buddha University, Gr. Noida, India 